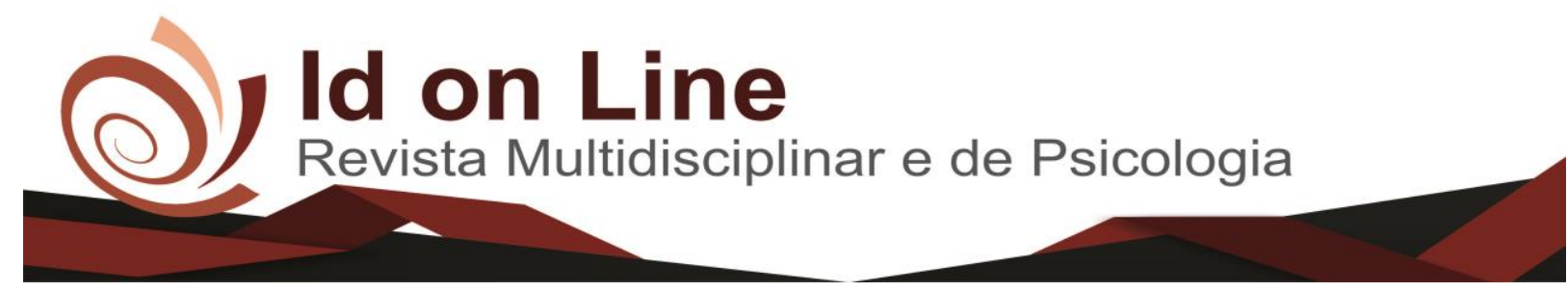

Artigo

\title{
Diagnósticos de Enfermagem Identificados em Pessoas Idosas com Diabetes mellitus
}

\author{
Nivaneide Ferreira Ramos de Sousa Lacerda ${ }^{l}$; Pollyanna Viana Lima ${ }^{2}$
}

\begin{abstract}
Resumo: O objetivo foi Identificar os diagnósticos de enfermagem em pessoas idosas com Diabetes mellitus atendido em uma Estratégia Saúde da Família. Metodologia: Trata-se de um estudo de casos múltiplos, realizado com 20 idosos portadores de Diabetes mellitus, realizado em setembro e outubro de 2017. Para a coleta de dados foi utilizado dois instrumentos: Questionário Sóciobiodemográfico e Econômico e Roteiro para anamnese e exame físico. O projeto obteve aprovação do Comitê de Ética em Pesquisa da Faculdade Independente do Nordeste, com parecer de aprovação $\mathrm{n}^{\mathrm{o}}$ 2.261.878. Resultados: Foram encontrados 14 diagnósticos diferentes, entre os mais evidenciados, destaca-se: risco de integridade da pele prejudicada (100\%); risco de lesão (100\%); risco para infecção (100\%); dor crônica (100\%); síndrome do idoso frágil (60\%); risco de trauma (50\%), acuidade visual prejudicada $(50 \%)$ e risco de quedas $(50 \%)$. Considerações Finais: Foi evidenciado que os idosos apresentam inúmeros problemas relacionados ao diabetes e são extremamente susceptíveis a complicações.
\end{abstract}

Palavras-chave: Idosos; Diabetes mellitus; Diagnósticos de Enfermagem; Complicações; Envelhecimento.

\section{Nursing Diagnosis Identified in Elderly People with Diabetes mellitus}

Abstract: Objective: To identify the nursing diagnoses in elderly people with Diabetes mellitus treated in a Family Health Strategy. Methodology: This is a multiple case study carried out with 20 elderly people with Diabetes mellitus, carried out in September and October 2017. Two instruments were used to collect data: SocioBiodemographic and Economic Questionnaire and Roadmap for anamnesis and physical examination. The project was approved by the Research Ethics Committee of the Faculdade Independente do Nordeste, with approval no. 2,261,878. Results: 14 different diagnoses were found, among the most evidenced: the risk of impaired skin integrity $(100 \%)$; risk of injury $(100 \%)$; risk for infection $(100 \%)$; chronic pain $(100 \%)$; syndrome of the frail elderly (60\%); risk of trauma (50\%), impaired visual acuity $(50 \%)$ and risk of falls $(50 \%)$. Final Thoughts: It has been shown that the elderly present numerous problems related to diabetes and are extremely susceptible to complications.

Keyword: Elderly; Diabetes mellitus; Nursing Diagnostics; Complications; Aging.

\footnotetext{
${ }^{1}$ Graduanda de Enfermagem pela Faculdade Independente do Nordeste. Professora de nível básico (Educação de Jovens e Adultos - EJA) na Rede Municipal de Ensino do Município de Cândido Sales.

${ }^{2}$ Mestre e Doutoranda do Programa de Pós-graduação Stricto Sensu em Memória:Linguagem e Sociedade da Universidade Estadual do Sudoeste da Bahia. Foi Bolsista em nível de Mestrado Acadêmico pela Fundação de Amparo à Pesquisa do Estado da Bahia - FAPESB. Bacharel em Enfermagem pela Faculdade Independente do Nordeste e Licenciada em Geografia pela Universidade Estadual do Sudoeste da Bahia. Contato: pollyanna@fainor.com.br.
} 


\section{Introdução}

Diversos estudos mostram que o aumento da população idosa é crescente e significativo, pois traz diversas implicações. Os estudos também mostram que esta tendência é relevante em todos os países (SDH, 2013; FREITAS; BARBOSA, 2015).

O processo de envelhecimento é único, gradual, individual e distinto entre todos os indivíduos, no entanto, traz algumas consequências comuns a todos, como por exemplo, o aumento das Doenças Crônicas Não Transmissíveis (DCNT). De acordo o Ministério da Saúde e dados do Sistema de Informação em Mortalidade (SIM), as DCNT são multifatoriais, e constituem um sério problema na atualidade, pois são responsáveis por cerca de $60 \%$ das mortes por ano (BRASIL, 2011; SIM, 2015).

Dentre as DCNT, o Diabetes mellitus (DM) merece destaque, devido seu crescimento nos últimos anos. Dados da International Diabetes Federation (IDF) revelaram que existam cerca de 26 milhões de indivíduos com Diabetes e é esperado o aumento deste número, devido o crescimento da população idosa (IDF, 2012). "O Diabetes mellitus destaca-se por sua alta prevalência mundial e seu potencial para o desenvolvimento de complicações crônicas e agudas, quando não tratada adequadamente" (GOMIDES et al., 2013, p. 890).

Diante desse panorama é extremamente importante que os profissionais de saúde, especialmente aqueles que trabalham na Atenção Básica $(\mathrm{AB})$ possam traçar estratégias de cuidados para estes indivíduos, com intuito de estimular a autonomia e auto cuidado. $\mathrm{O}$ profissional de enfermagem, por meio da consulta de enfermagem e aplicação da Sistematização da Assistência de Enfermagem (SAE) tem a capacidade da detecção de problemas ou fatores que estejam interferindo no processo de adesão ao tratamento, ou na autonomia e autocuidado (MOURA et al., 2014; BRITO; NÓBREGA; SANTOS, 2013).

O levantamento de diagnósticos é a segunda etapa do PE, e consiste analisar os problemas identificados anteriormente e interpretá-los criteriosamente, para posterior análise serem agrupados em categorias próximas. Normalmente utiliza-se a taxonomia North American Nursing Diagnosis Association (NANDA), esta se trata de um instrumento multiaxial em que os diagnósticos são organizados em domínios (TANNURE; GONÇALVES, 2010; NANDA, 2013).

Diante disso, salienta-se a importância de estudos que avaliem idosos portadores de DM, através de uma anamnese e exame físico qualificada, para que posteriormente, por meio de 
diagnósticos de enfermagem possibilite a construção de um plano de cuidados que seja satisfatório e atenda as individualidades dos idosos.

Desta forma, este estudo objetivou identificar os diagnósticos de Enfermagem de idosos portadores de Diabetes mellitus atendido em uma Estratégia Saúde da Família.

\section{Metodologia}

Trata-se de um estudo de casos múltiplos realizado com idosos portadores de Diabetes mellitus. O estudo de casos múltiplos é um método eficaz como estratégia de pesquisa para investigar fenômenos relacionados à saúde e enfermagem, isso porque possibilita a captação de diferentes pontos de vista, aspectos objetivos e subjetivos presentes em uma situação de saúde em seus contextos social e cultural (ALVES-MAZZOTTI, 2006).

A pesquisa foi realizada em uma Equipe de Saúde da Família, do Município de Cândido Sales - BA, entre setembro e outubro de 2017.

Os indivíduos participantes do estudo foram 20 idosos portadores de Diabetes mellitus, cadastrados na referida Unidade Básica de Saúde. Ressalta-se que a escolha destes participantes aconteceu de forma aleatória, considerando aqueles que comparecerem no dia da consulta mensal para recebimento da medicação.

Para a coleta de dados foi utilizado um questionário Sóciobiodemográfico e Econômico, um Roteiro para anamnese e exame físico de enfermagem.

O perfil sociobiodemográfico e econômico da população do estudo foi caracterizado e avaliado através do Questionário Sociodemográfico e econômico, elaborado pelos próprios pesquisadores, o qual abordou questões sobre sexo do idoso, idade, cor, religião, grau de escolaridade, estado civil e renda mensal, doenças associadas, uso de medicamento, atividade física, entre outros.

A anamnese e exame o físico constituem parte do processo de enfermagem e segundo Tannure e Gonçalves (2010), são suficientes para definir o diagnóstico e o manejo do caso. Além dos questionamentos e do exame físico, é imprescindível que as anotações sejam realizadas de maneira clara e compreensível. Para realização da Anamnese, foi utilizado um roteiro elaborado pelos pesquisadores com questões relativas ao histórico de saúde, história da doença atual, resultados de exames, medicamentos de uso atual, antecedentes clínicos pessoais, 
antecedentes clínicos familiares, alergias, determinantes sociais, condições socioeconômicas, culturais, ambientais gerais e estilo de vida dos idosos. Já para a coleta de dados do exame físico, foi utilizado um roteiro de exame físico, também elaborado pelos pesquisadores, seguindo o exame céfalo-podálico com aspectos gerais, cabeça e pescoço, tórax e abdome, Membros Superiores e Membros Inferiores (MMSS/MMII) e sinais vitais.

A análise dos dados foi realizada utilizando-se o pacote estatístico SPSS®, versão 22.0. Para todos os procedimentos, foi adotado um intervalo de confiança de $5 \%(\mathrm{p}<0,05)$. Das informações obtidas a partir da anamnese e exame físico foram levantados os diagnósticos de enfermagem.

O projeto foi encaminhado a Secretaria de Saúde do município de Cândido Sales - BA, para apreciação. Após resposta positiva o mesmo foi encaminhado ao Comitê de Ética em Pesquisa (CEP) da Faculdade Independente do Nordeste (FAINOR), com Certificado de Apresentação para Apreciação Ética (CAAE) no 70115617.4.0000.5578 e com parecer de aprovação $n^{\circ} 2.261 .878$.

A pesquisa obedeceu todos os preceitos da Resolução 466/12, onde os participantes da pesquisa foram respeitados em sua dignidade e autonomia, reconhecendo sua vulnerabilidade, assegurando sua vontade sob forma de manifestação expressa, livre e esclarecida, de contribuir e permanecer ou não na pesquisa. Os participantes da pesquisa foram devidamente orientados quanto aos objetivos, ficando os mesmo livres para participarem ou não. Os indivíduos que aceitaram participar assinaram um Termo de Consentimento Livre e Esclarecido (TCLE).

\section{Resultados}

A tabela 1 apresenta o perfil sóciobiodemográfico dos participantes da pesquisa.

Participaram do estudo 20 idosos, com faixa etária entre de 60 e 88 anos, com média de 69,3 anos. Em sua maioria mulheres $(75 \%)$, de cor parda (65\%), católicos (70\%), com nenhuma escolaridade (45\%), casados (45\%), aposentados com renda de 1 salário mínimo (80\%).

Dos idosos participantes do estudo, $25 \%$ fazem uso de medicações associadas: Metformina $850 \mathrm{mg}$ + Glibenclamida $5 \mathrm{mg}$ e $10 \%$ Glibenclamida $5 \mathrm{mg}$ + Insulina.

Quanto às doenças associadas ao diabetes as únicas descritas foram: Hipertensão Arterial Sistêmica (30\%) e Doenças Cardiovasculares (5\%). 
Tabela 1 - Distribuição de idosos quanto ao perfil sóciobiodemográfico e econômico, Cândido Sales - Bahia, 2017.

\begin{tabular}{|c|c|c|}
\hline Variável & $\mathbf{N}$ & $\%$ \\
\hline \multicolumn{3}{|l|}{ Sexo } \\
\hline Masculino & 5 & 25,00 \\
\hline Feminino & 15 & 75,00 \\
\hline \multicolumn{3}{|l|}{ Cor/Raça } \\
\hline Parda & 13 & 65,00 \\
\hline Branca & 6 & 30,00 \\
\hline Negra & 1 & 5,00 \\
\hline \multicolumn{3}{|l|}{ Religião } \\
\hline Católicos & 14 & 70,00 \\
\hline Protestantes & 6 & 30,00 \\
\hline \multicolumn{3}{|l|}{ Escolaridade } \\
\hline Nenhuma escolaridade & 9 & 45,00 \\
\hline Ensino fundamental & 10 & 50,00 \\
\hline Pós-graduada & 1 & 5,00 \\
\hline \multicolumn{3}{|l|}{ Estado Civil } \\
\hline Casados & 9 & 45,00 \\
\hline Viúvos & 6 & 30,00 \\
\hline Divorciado & 1 & 5,00 \\
\hline Nunca casaram & 4 & 20,00 \\
\hline \multicolumn{3}{|l|}{ Renda mensal } \\
\hline Aposentadoria de 1 salário mínimo & 16 & 80,00 \\
\hline Sem renda mensal & 2 & 10,00 \\
\hline Pensão de 1 salário mínimo & 1 & 5,00 \\
\hline Trabalho com renda de 1 salário mínimo & 1 & 5,00 \\
\hline \multicolumn{3}{|l|}{ Medicamentos } \\
\hline Metformina 850mg+ Glibenclamida 5mg & 5 & 25,00 \\
\hline Glibenclamida 5mg + Insulina & 2 & 10,00 \\
\hline \multicolumn{3}{|l|}{ Doenças associadas } \\
\hline $\mathrm{DM}+\mathrm{HAS}$ & 6 & 30,00 \\
\hline $\mathrm{DM}+\mathrm{DCV}$ & 1 & 5,00 \\
\hline \multicolumn{3}{|l|}{ Complicações } \\
\hline Pé diabético & 3 & 15,00 \\
\hline Doença cardiovascular & 1 & 5,00 \\
\hline Amputações & 3 & 15,00 \\
\hline Retinopatia diabética & 10 & 50,00 \\
\hline Cegueira total & 1 & 5,00 \\
\hline \multicolumn{3}{|l|}{ Queixas } \\
\hline Dor lombar & 6 & 30,00 \\
\hline Fraqueza & 7 & 35,00 \\
\hline Prurido & 5 & 25,00 \\
\hline Fissuras calcâneas & 5 & 25,00 \\
\hline \multicolumn{3}{|l|}{ Praticam Atividades Físicas? } \\
\hline Sim & 0 & 0,00 \\
\hline Não & 20 & 100,00 \\
\hline
\end{tabular}

Fonte: Dados da pesquisa 
Em relação às complicações decorrentes do Diabetes, o estudo encontrou: Retinopatia Diabética (50\%), pé diabético (15\%), Amputações (15\%) e Cegueira total (5\%).

No que se refere à atividade física, $100 \%$ dos idosos não fazem nenhum tipo. Já às queixas mais frequentes referidas pelos idosos, à fraqueza (35\%), a dor lombar (30\%), o prurido $(25 \%)$ e fissuras calcâneas $(25 \%)$ foram as mais evidenciadas.

Quanto aos diagnósticos de enfermagem baseados na Taxonomia North American Nursing Diagnosis Association (NANDA), os mesmos estão dispostos na tabela 2 baseia nas definições dos diagnósticos.

Tabela 2 - Distribuição percentual da população idosa de acordo os diagnósticos de enfermagem realizados pela taxonomia NANDA. Cândido Sales - BA, 2017.

\begin{tabular}{lcc}
\hline Variáveis & $\mathbf{N}$ & $\mathbf{\%}$ \\
\hline Risco de integridade da pele prejudicada & 20 & 100,00 \\
Dor Crônica & 20 & 100,00 \\
Risco de lesão & 20 & 100,00 \\
Risco para infecção & 20 & 100,00 \\
Síndrome do idoso frágil & 12 & 60,00 \\
Acuidade visual prejudicada & 10 & 50,00 \\
Risco de quedas & 10 & 50,00 \\
Risco trauma & 10 & 50,00 \\
Risco de síndrome do idoso frágil & 8 & 40,00 \\
Deambulação prejudicada & 6 & 30,00 \\
Mobilidade física prejudicada & 5 & 25,00 \\
Integridade da pele prejudicada & 4 & 20,00 \\
Comunicação verbal prejudicada & 1 & 5,00 \\
Interação social prejudicada & 1 & 5,00 \\
\hline TOTAL & 20 & 100,00 \\
\hline
\end{tabular}

Fonte: Dados da pesquisa

Foram encontrados 14 diagnósticos diferentes, a saber: risco trauma (50\%), integridade da pele prejudicada (20\%), risco de integridade da pele prejudicada (100\%), comunicação verbal prejudicada (5\%), interação social prejudicada (5\%), risco para infecção (100\%), mobilidade física prejudicada (25\%), acuidade visual prejudicada (50\%), deambulação prejudicada (30\%), dor crônica (100\%), risco de lesão (100\%), síndrome do idoso frágil (60\%), risco de síndrome do idoso frágil (40\%), risco de quedas (50\%). 


\section{Discussão}

No que tange aos aspectos sociobiodemográficos e econômicos, observa-se que os idosos apresentam tendências semelhantes a outros estudos, como o de Lopes et al. (2014) e o de Nogueira et al. (2015). Estes estudos mostram que os idosos são na maioria aposentados, analfabetos, com renda mensal de um salário mínimo e são portadores de doenças crônicas, especialmente o diabetes associado à hipertensão e doenças cardiovasculares.

Sabe-se que a baixa escolaridade interfere especialmente em ações de autocuidado e entendimento acerca da própria doença, levando a maior atenção por parte dos profissionais no que tange à compreensão dos idosos em relação às orientações e informações dadas. Com relação à renda mensal dos idosos, a baixa renda mostrada, associada aos demais indicadores refletem nas desigualdades sociais de forma direta (LOPES et al., 2014; NOGUEIRA et al., 2015).

Para Nogueira et al. (2015), nessa trama de desigualdades sociais, tem repercussão, tanto nos padrões de vida e de doenças, quanto no acesso e utilização dos serviços de saúde. O autor ainda cita que todos os indicadores a cima contribuem diretamente na qualidade de vida dos idosos.

Em relação às medicações utilizadas para tratamento do DM, observa-se que a maioria faz uso de Metformina 850mg + glibenclamida $5 \mathrm{mg}$ e uma minoria também faz o uso da insulina. Todos os medicamentos fazem parte da farmácia básica da Unidade de Saúde e devem ser acessíveis aos pacientes (SBD, 2016).

O objetivo primordial do tratamento antidiabético é a realização do controle glicêmico e melhoria da qualidade de vida. Para além de medicamentos, a educação em saúde deve ser uma premissa, pois provoca mudanças, reduz barreiras e promove a autonomia das pessoas com diabetes (SBD, 2016, p. 223).

Nogueira et al. (2015) aponta em seu estudo que a capacidade do DM de causar variadas alterações na saúde dos pacientes, afeta a vida cotidiana dos mesmos, fazendo com que eles temam a doença e por consequente, causando diminuição da qualidade de vida, o que reforça mais uma vez a necessidade de orientações quanto a doença e autocuidado (NOGUEIRA et al., 2015).

No tocante à associação DM e HAS, o estudo mostrou que a associação de Diabetes mellitus e Hipertensão Arterial Sistêmica, está presente em uma parcela significativa dos idosos, tendência que é observada em diversos outros estudos e órgãos no Brasil e ao redor do mundo. 
A alta prevalência das duas doenças associadas se deve, especialmente, por serem DCNTs multifatoriais, com longo período de desenvolvimento e longa duração. Mas também, devido à associação está muitas vezes relacionadas a outros fatores de risco como o tabaco, o sedentarismo, alimentação inadequada e consumo nocivo de álcool (BRASIL, 2011, 2013; ALENCAR et al., 2014).

Destaca-se que pessoas com DM, que são também portadores de HAS têm mais fatores de risco para terem complicações, bem como para desenvolver acidente vascular encefálico, doença renal, doença cardíaca coronariana e insuficiência cardíaca (CAPELETTI; SALLA, 2016). Portanto, a vigilância constante deve ser a premissa das equipes de saúde.

No presente estudo, observou-se que a inatividade física estava presente em todos os idosos. A falta de atividades físicas contribui para a aquisição das DCNTs, como também para complicação das mesmas. Um estudo realizado por Gomides et al. (2013), com idosos diabéticos, mostrou que a realização de atividades físicas era inexistente no grupo de indivíduos pesquisados, resultado semelhante ao aqui encontrado.

É importante o estímulo às praticas de atividade física, uma vez que estas contribuem de forma positiva para a saúde de idosos com DM, com melhora das condições clínicas e da capacidade funcional e da qualidade de vida (FRACHI et al., 2008; GOMIDES et al., 2013; SBD, 2016).

Outro dado relevante encontrado neste estudo refere-se à presença de complicações decorrentes dos DM. Dentre as mais citadas destaca-se: retinopatia diabética $(50 \%)$ e pé diabético, com consequente amputação (15\%).

Estima-se que $99 \%$ dos indivíduos com DM do tipo 1 e $60 \%$ de pessoas com DM do tipo 2 progridem para alguma forma de Retinopatia Diabética (RD) após 20 anos com a doença. A RD tem sido descrita como uma complicação microvascular do diabetes, associada à longa duração da doença e ao controle glicêmico impróprio (ALVES et al., 2014), sendo também a principal causa de cegueira nos indivíduos. Ressalta-se que metade dos indivíduos do presente estudo apresentavam a retinopatia diabética e 5\% já estavam com cegueira total (ALVES et al., 2014).

O pé diabético foi também outra complicação muito evidenciada neste estudo. De acordo com Gomides et al. (2013) os pacientes reconhecem que o autocuidado com os pés, na perspectiva de evitar complicações, deve existir, no entanto, ele não é feito de maneira eficaz, pois as complicações ainda existem. O pé diabético é um problema grave de saúde pública, pois traz grande impacto socioeconômico devido ao tratamento. Além disso, gera ao portador da 
condição longo tempo de internamento, internações recorrentes, incapacidades físicas, sociais, afastamento do trabalho e de algumas atividades cotidianas (ROMUALDO; VASCONCELOS; SOUZA, 2015).

No que tange aos diagnósticos de enfermagem levantados, o risco de lesão estava presente em todos os idosos avaliados e é definido como: "Resultado de condições ambientais interagindo com os recursos adaptativos e defensivos do indivíduo" (NANDA, 2017, p. 497). O Risco de integridade da pele prejudicada, também presente em todos os idosos é definido como: "Risco de epiderme e/ou derme alteradas" (NANDA, 2017, p. 495).

De acordo Alencar et al. (2014), o risco de lesão e o risco de integridade da pele prejudicada, estão associados a presença de neuropatia diabética, condição presente em muitos portadores de DM tipo 2. Nesta condição, o indivíduo perde a sensibilidade de periferias, como tato, sensação térmica e sensação de dor, consequentemente há retardo na diferença para identificação de calor e frio e dor, deixando o indivíduo suscetível à aquisição de lesões na maioria das vezes complexas e podem levar ao acometimento do membro inferior, causando amputação.

É extremamente relevante que o profissional de enfermagem, saiba avaliar os membros inferiores dos pacientes e orientá-los no intuito do diagnóstico precoce de alterações que possam levar a acometimentos futuros e danosos (ALENCAR et al., 2014). Além do diagnóstico de risco de integridade, também foi identificado o diagnóstico efetivo da Integridade da pele prejudicada em alguns idosos avaliados. A integridade da pele prejudicada ocorre especialmente nos membros inferiores dos idosos, por meio de úlceras infectadas ou úlceras venosas (ROMUALDO; VASCONCELOS; SOUZA, 2015).

Já o risco de infecção também presente em todos os idosos é definido como "Risco de ser invadido por organismos patogênicos" (NANDA, 2015, p. 485), e é muito comum em idosos diabéticos, pois devido à disfunção endotelial, os mesmos tornam-se susceptíveis a infecções.

As infecções em pacientes diabéticos devem ser observadas cuidadosamente, uma vez que elas podem levar a complicações maiores como: amputações de membros, maior número de retornos nos serviços de saúde e consequentemente maiores custos ao sistema de saúde (IDF, 2012).

No que se refere aos diagnósticos de Dor crônica, presente também em todos os idosos participantes deste estudo, esta é definida como: "Experiência sensorial e emocional desagradável que surge de lesão tissular real ou potencial ou descrita em termos de tal lesão (Associação Internacional para o Estudo da Dor); início súbito ou lento, de intensidade leve a 
intensa, constante ou recorrente, sem um término antecipado ou previsível e com uma duração de mais de seis meses" (NANDA, 2017, p. 549).

A dor crônica ocorre nos idosos diabéticos devido incapacidade funcional, neste sentido, Franchi et al. (2008), mostrou em seu estudo que o envelhecimento ativo proporciona benefícios para o corpo, dentre ele cita-se a melhoria e diminuição das dores crônicas e dores articulares, além da diminuição de taxa metabólica e melhoria da composição corporal equilíbrio entre gordura e massa magra.

A Síndrome do Idoso Frágil foi um dos diagnósticos mais evidenciados neste estudo. De acordo Lana e Schneider (2014) a Síndrome do Idoso Frágil é caracterizada por uma série de alterações biológicas decorrentes do envelhecimento patológico, devido diminuição da homeostase e consequente redução da capacidade de adaptação do organismo humano as adversidades, levando a declínios nos diversos sistemas fisiológicos.

Dentre os sinais e sintomas da Síndrome do Idoso Frágil estão: "perda de peso não intencional (5kg nos últimos cinco anos), autorrelato de fadiga, diminuição da força de preensão, redução das atividades físicas, diminuição na velocidade da marcha (lentidão) e diminuição das relações sociais" (LANA; SCHNEIDER, 2014, p. 674). Destaca-se ainda que além dos diagnósticos de Síndrome do Idoso Frágil, ocorreu também o Risco de Síndrome de idoso Frágil em um número significativo de idosos avaliados.

A acuidade visual prejudicada foi um diagnóstico de enfermagem presente em metade dos idosos avaliados. Alves et al. (2014) afirma que a presença de alterações oftalmológicas em pacientes portadores de DM e HAS é extremamente alta e significativa. Estes mesmos autores realizaram um estudo observacional, composto por idosos portadores de DM e HAS e as alterações oftalmológicas estavam presentes em $39,1 \%$ dos idosos diabéticos, sendo que a população com diabetes era de $45,5 \%$, percentual extremamente alto. Os autores ainda trazem que se houvessem oftalmoscópios disponíveis nas Unidades Básicas de Saúde, a identificação precoce de alterações oculares seria favorecida, prevenindo complicações futuras que podem causar a cegueira ou diminuição permanente da acuidade visual (ALVES et al., 2014).

O Risco de quedas, presente também em metade dos idosos é definido como: "Risco de suscetibilidade aumentada para quedas que podem causar dano físico" (NANDA, 2017, p. 505). De acordo D'Alencar e D'Alencar (2013), dentre os fatores constituintes para o risco de queda estão os distúrbios na marcha e equilíbrio e apesar de nem todas as quedas resultarem em lesões ou danos, cerca de $2 / 3$ da mortalidade acidental dos idosos é causada devido quedas, 
especialmente em idosos maiores de 65 anos. Ressalta-se ainda que as lesões e os danos constituem assim, como o pé diabético, um problema de saúde pública.

O Risco de traumas, presente em metade dos idosos avaliados é definido como: "Risco de lesão tecidual acidental (p. ex., ferida, queimadura, fratura)" (NANDA, 2017, p. 513), assim como o risco de lesão e o risco de integridade da pele prejudicada, ocorre devido complicações, especialmente devido à neuropatia diabética, com diminuição dos reflexos e sensibilidade protetora, ocasionando maior susceptibilidade (BRASIL, 2013; ALENCAR et al., 2014).

Ressalta-se ainda a identificação de diagnósticos menos recorrentes como: Deambulação prejudicada, definida como: "Limitação à movimentação independente, a pé, pelo ambiente" (NANDA, 2017, p. 280). Mobilidade física prejudicada caracterizada como "Limitação no movimento físico independente e voluntário do corpo ou de uma ou mais extremidades" (NANDA, 2017, p. 283). E por fim, porém não menos importante, foi encontrado o diagnóstico de Comunicação verbal prejudicada, definido como "Habilidade diminuída, retardada ou ausente para receber, processar, transmitir e/ou usar um sistema de símbolos" (NANDA, 2017, p.335) e Interação social prejudicada que é definida como a "Quantidade insuficiente ou excessiva, ou qualidade ineficaz, de troca social" (NANDA, 2017, p. 379).

\section{Considerações Finais}

Este estudo buscou identificar os diagnósticos de Enfermagem de idosos portadores de Diabetes mellitus atendido em uma Estratégia Saúde da Família e chegou à conclusão de que os idosos apresentam inúmeros problemas relacionados ao diabetes, o que gerou o levantamento de um quantitativo relativamente grande de diagnósticos de enfermagem.

Além disso, foi evidenciado que os idosos são extremamente susceptíveis a complicações, haja vista, a presença de fatores de riscos como o sedentarismo e a presença de diversas complicações associadas ao diabetes, especialmente a retinopatia diabética e amputações.

Dentre as limitações do estudo ressaltam-se as dificuldades de acesso aos idosos, o que resultou em pequeno quantitativo de participantes, porém acredita-se que é um estudo significativo, pois traz uma realidade preocupante, que precisa de intervenções urgentes. Além disso, apresenta uma contribuição valorosa para a área acadêmica e para a sociedade no que 
tange a suscitar discussões na área e a possibilidade do desenvolvimento de novas políticas de saúde para este grupo populacional. Por fim, sugere-se a realização de estudos transversais que possam abranger um grupo populacional maior.

\section{Referências}

ALENCAR, L. L. et al. Perfil epidemiológico de idosos com Diabetes mellitus tipo 2 cadastrados na Estratégia Saúde da Família. Revista Eletrônica Gestão \& Saúde, Saúde do Idoso, p.2972-89, 2014.

ALVES A. P. et al. Retinopatia em pacientes hipertensos e/ou diabéticos em uma unidade de saúde da família. Rev Bras Oftalmol., v. 73, n. 2, p. 108-11, 2014.

ALVES-MAZZOTTI, A. J. Usos e abusos dos estudos de caso. Cadernos de Pesquisa, v. 36, n. 129, p. 637-651, 2006.

BRASIL. Ministério da Saúde. Secretaria de Vigilância em Saúde. Departamento de Análise de Situação de Saúde. Plano de ações estratégicas para o enfrentamento das doenças crônicas não transmissíveis (DCNT) no Brasil 2011-2022. Brasília, DF: MS, 2011.

BRASIL. Ministério da Saúde. Secretaria de Atenção à Saúde. Departamento de Atenção Básica. Estratégias para o cuidado da pessoa com doença crônica: Diabetes mellitus. Brasília, DF: MS, 2013.

BRITO, S. S.; NÓBREGA, R. V.; SANTOS, S. R. dos et al. Sistematização da Assistência de Enfermagem na atenção básica aos hipertensos: Relato de experiência. Rev enferm UFPE online., v. 7, n. 8, p.5345-50, 2013. Disponível em:

<https://periodicos.ufpe.br/revistas/revistaenfermagem/article/download/11812/14203 >. Acesso em: 15 out. 2017.

CAPELETTI1, A. P.; SALLA, L. F. Relação entre hipertensão arterial sistêmica e diabetes mellitus tipo 2. In: CONGRESSO GAÚCHO DE CLÍNICA MÉDICA, 13., 2016. Anais... v. 2, n. 7. 2016. Disponível em: <http://pdf.blucher.com.br.s3-sa-east1.amazonaws.com/medicalproceedings/xiii-cgcm/1457142812.pdf >. Acesso em: 11 out. 2017.

D'ALENCAR, M. S.; D'ALENCAR, F. S. Quedas na Velhice: uma ocorrência impeditiva à manutenção da funcionalidade. Memorialidades. v. único, n. 20, p. 9-27, 2013.

FRANCHI, K. M. B. et al. Capacidade funcional e atividade física de idosos com Diabetes Tipo 2. Revista Brasileira de Atividade Física \& Saúde, v. 13, n. 3, p. 158-166, 2008. 
FREITAS, C. E.; BARBOSA, R. R. A previdência social e as distorções na distribuição de renda. Pesquisa \& debate, v. 26, n. 1(47), p. 173-197, 2015.

GOMIDES, D. S. et al. Autocuidado das pessoas com diabetes mellitus que possuem complicações em membros inferiores. Acta Paul Enferm., v. 26, n. 3, p. 289-93, 2013.

INTERNATIONAL DIABETES FEDERATION - IDF. Global Guideline for Type 2 Diabetes. University of Sydney. Sydney, Australia, 2012.

LANA, L. D.; SCHNEIDER, R. H. Síndrome de fragilidade no idoso: uma revisão narrativa. Rev. Bras. Geriatr. Gerontol., v. 17, n. 3, p. 673-680, 2014.

LOPES, F. A. M. et al. Perfil epidemiológico em idosos assistidos pela Estratégia Saúde da Família. REAS v.3, n.1, p. 84-94, 2014. Disponível em:

<http://uftm.edu.br/revistaeletronica/index.php/enfer/article/view/933>. Acesso em: 11 out. 2017.

MOURA, D. J. M. et al. Sistematização da assistência de enfermagem fundamentada na CIPE® e na teoria da adaptação em hipertensos. Rev. Eletr. Enf. v. 16, n. 4, p.710-9, 2014. Disponível em: <www.fen.ufg.br/revista/v16/n4/pdf/v16n4a02.pdf >. Acesso em: 11 out. 2017.

NANDA International. Diagnósticos de enfermagem da NANDA: definições e classificação 2012-2014. Tradução Regina Machado Garcez; revisão técnica: Alba Lucia Bottura Leite de Barros ... [et al.]. Dados eletrônicos. Porto Alegre: Artmed, 2013.

NANDA International, Inc. Nursing Diagnoses: definitions \& classification 2015-2017, Tenth Edition. Edited by T. Heather Herdman and Shigemi Kamitsuru. by John Wiley \& Sons. NANDA International, Inc. Published, 2014.

NANDA International. Diagnósticos de enfermagem da NANDA: definições e classificação 2015-2017. Dados eletrônicos. Tradução Regina Machado Garcez; revisão técnica Alba Lucia Bottura Leite de Barros et al. Porto Alegre: Artmed, 2015.

NOGUEIRA, M. F. et al. Avaliação da Qualidade de Vida de idosos com Diabetes Mellitus. Rev enferm UFPE on line., n. 9, v.(Supl. 6), p. 8688-97, 2015.

ROMUALDO, S. H.; VASCONCELOS, T. L. S.; SOUZA, F. S. Prevenção e cuidado do pé diabético: uma questão de saúde pública, sob a visão da enfermagem. Rev educação, meio ambiente e saúde, v. 2 n. 16, p. 134-154, 2015.

SECRETARIA DE DIREITOS HUMANOS - SDH. Secretaria nacional de promoção defesa dos direitos humanos. 2013. Disponível em: <http://www.sdh.gov.br/assuntos/pessoaidosa/dados-estatisticos/DadossobreoenvelhecimentonoBrasil.pdf>. Acesso em: 07 set. 2017.

SISTEMA DE INFORMAÇÃO EM MORTALIDADE - SIM, 2015. Disponível em: $<$ http://portalsaude.saude.gov.br/index.php/o-ministerio/principal/secretarias/svs/mortalidade >. Acesso em: 07 set. 2017. 
SOCIEDADE BRASILEIRA DE DIABETES - SBD. Diretrizes da sociedade brasileira de diabetes (2015-2016). Organização José Egidio Paulo de Oliveira, Sérgio Vencio. São Paulo: A.C. Farmacêutica, 2016.

TANNURE, M. C.; GONÇALVES, A. M. P. Sistematização da Assistência de Enfermagem: guia prático. Guanabara Koogan, 2010.

\section{Como citar este artigo (Formato ABNT):}

LACERDA, Nivaneide F.R de S.; LIMA, Pollyanna V. Diagnósticos de Enfermagem Identificados em Pessoas Idosas com Diabetes mellitus. Id on Line Revista Multidisciplinar e de Psicologia, 2017, vol.11, n.38, p. 431-444. ISSN: 1981-1179.

Recebido: 30.10 .2017

Aceito: 01.11 .2017 